\title{
CORRIGENDUM AND ADDENDUM TO 'LINEARLY RECURRENT SUBSHIFTS HAVE A FINITE NUMBER OF NON-PERIODIC FACTORS'
}

\author{
FABIEN DURAND
}

\begin{abstract}
We prove that a subshift $(X, T)$ is linearly recurrent if and only if it is a primitive and proper $S$-adic subshift. This corrects Proposition 6 in F. Durand (Ergod. Th. \& Dynam. Sys. 20 (2000), 1061-1078).
\end{abstract}

\section{INTRODUCTION AND DEFINITIONS}

In this paper we freely use the definitions and the notations of $\mathrm{Du}$. Proposition 6 in $[\mathrm{Du}]$ is false: There exist primitive $S$-adic subshifts that are not linearly recurrent (LR). We will give an example. Nevertheless the other part of this proposition is true: If a subshift is LR then it is primitive $S$-adic.

The author apologizes for the mistake. We correct this proposition with the next one, but before we have to give and to recall some definitions. Let $A$ and $B$ be two finite alphabets.

Let $x$ be an element of $A^{\mathbb{N}}$ or $A^{\mathbb{Z}}$. We call occurrence of $u \in A^{*}$ in $x$ every integer $i$ such that $x_{[i, i+|u|-1]}=x_{i} x_{i+1} \ldots x_{i+|u|-1}=u$. A return word to $u \in A^{*}$ in $x$ is a word $w$ such that $w u$ has an occurrence in $x, u$ is a prefix of $w u$ and $u$ has exactly 2 occurrences in $w u$. We say that $x$ is linearly recurrent $(\mathrm{LR})$ (with constant $K \in \mathbb{N}$ ) if it is uniformly recurrent and if for all $u$ having an occurrence in $x$ and all return words, $w$, to $u$ in $x$ we have $|w| \leq K|u|$.

Let $T$ be the shift transformation defined on $A^{\mathbb{Z}}$. We say the subshift $(X, T)$ is generated by $x$ if $X$ is the set of the sequences $z$ such that $z_{[i, j]}$ has an occurrence in $x$ for all intervals $[i, j] \subset \mathbb{Z}$. The subshift $(X, T)$ is linearly recurrent if it is minimal and contains a LR sequence. We remark that if $x \in A^{\mathbb{Z}}$ is linearly recurrent then $x$ and $x_{[0,+\infty)}$ generate the same LR subshift.

Let $a$ be a letter of $A, S$ a finite set of morphisms $\sigma$ from $A(\sigma) \subset A$ to $A^{*}$ and $\left(\sigma_{n}: A_{n+1} \rightarrow A_{n}^{*} ; n \in \mathbb{N}\right)$ be a sequence of $S^{\mathbb{N}}$ such that $\left(\sigma_{0} \sigma_{1} \cdots \sigma_{n}(a a \cdots) ; n \in \mathbb{N}\right)$ converges in $A^{\mathbb{N}}$ to $x$. We will say that $x$ is a $S$-adic sequence on $A$ (generated by $\left(\sigma_{i} ; i \in \mathbb{N}\right) \in S^{\mathbb{N}}$ and $a$ ). If there exists an integer $s_{0}$ such that for all nonnegative integers $r$ and all $b \in A_{r}$ and $c \in A_{r+s_{0}+1}$, the letter $b$ has an occurrence in $\sigma_{r+1} \sigma_{r+2} \cdots \sigma_{r+s_{0}}(c)$, then we say that $x$ is a primitive $S$-adic sequence (with constant $\left.s_{0}\right)$.

Let $\sigma: A \rightarrow B^{*}$ be a morphism. We say $\sigma$ is proper if there exist two letters $r, l \in B$ such that for all $a \in A$ the first letter of $\sigma(a)$ is $l$ and the last letter of $\sigma(a)$ is $r$. We say the sequence $x \in A^{\mathbb{N}}$ is a proper $S$-adic sequence if it is a $S$-adic sequence and the morphisms in $S$ are proper. The subshift generated by a proper $S$-adic sequence is called proper $S$-adic subshift. 
Proposition 1.1. The subshift $(X, T)$ is $L R$ if and only if it is a primitive and proper $S$-adic subshift.

\section{Counterexample to the Proposition 6 of $\mathrm{Du}$}

In this section we give a counterexample to Proposition 6 in $[\mathrm{Du}$, i.e., a primitive $S$-adic subshifts that is not LR.

Let $A=\{a, b, c\}$ be an alphabet, and, $\sigma: A \rightarrow A^{*}$ and $\tau: A \rightarrow A^{*}$ be two morphisms defined by

$$
\begin{aligned}
\sigma(a) & =a c b, & \tau(a) & =a b c \\
\sigma(b) & =b a b, & \tau(b) & =a c b, \\
\sigma(c) & =c b c, & \tau(c) & =a a c .
\end{aligned}
$$

We call $\mathcal{M}$ the set of all finite composition of elements of $S=\{\sigma, \tau\}$. For each element $\rho$ of $\mathcal{M}$ there exists a unique $n \in \mathbb{N}$ such that $|\rho(a)|=|\rho(b)|=|\rho(c)|=3^{n}$. We set $|\rho|=3^{n}$. We first give a lemma which proof is left to the reader.

Lemma 2.1. Let $z \in A^{\mathbb{N}}$ and $n \in \mathbb{N}$. The difference between two successive occurrences of the word ca in $\sigma^{n} \tau(z)$ is greater than $3^{n+1}$.

Let $x \in A^{\mathbb{N}}$ be the primitive $S$-adic sequence defined by

$$
x=\lim _{n \rightarrow+\infty} \sigma \tau \sigma^{2} \tau \cdots \sigma^{n} \tau(\text { aaa } \ldots) .
$$

We show $x$ is not LR. Let $n$ be an integer, we set $\rho_{n}=\sigma \tau \sigma^{2} \tau \cdots \sigma^{n} \tau$ and $y=$ $\lim _{l \rightarrow+\infty} \sigma^{n+2} \tau \sigma^{n+3} \tau \cdots \sigma^{n+l} \tau($ aaa $\ldots)$. We have $x=\rho_{n} \sigma^{n+1} \tau(y)$.

Since $\sigma(a)$ is a prefix of $y, \tau \sigma(a)=a b c a a c a c b, \sigma^{n+1}(a)=a u$ and $\sigma^{n+1}(c)=v c$, for some $u, v \in A^{*}$, then $c a$ appears in $\sigma^{n+1} \tau(y)$. Let $w$ be a return word to $c a$ in $\sigma^{n+1} \tau(y)$. Hence $w c a\left(=c a w^{\prime} c a\right.$ for some $\left.w^{\prime}\right)$ appears in $x$. The word $\rho_{n}(c a)$ appears exactly twice in $\rho_{n}\left(c a w^{\prime} c a\right)$ (the proof of this fact is left to the reader) hence $\rho_{n}(w)$ is a return word to $\rho_{n}(c a)$ in $x$.

Moreover from Lemma 2.1 we have $|w| \geq 3^{n+2}$. It implies $x$ is not LR because

$$
\frac{\left|\rho_{n}(w)\right|}{\left|\rho_{n}(c a)\right|}=\frac{|w|\left|\rho_{n}\right|}{2\left|\rho_{n}\right|} \geq \frac{3^{n+2}}{2}
$$

Let $z \in B^{\mathbb{N}}$ where $B$ is a finite alphabet. We denote by $L(z)$ the set of all words having an occurrence in $z$. For all $n \in \mathbb{N}$ we define $p_{z}(n)$ to be the number of distinct words of length $n$ in $L(z)$. In $[\mathrm{Du}]$ it is proved that if $z$ is LR with constant $K$ then $p_{z}(n) \leq K n$ for all $n \in \mathbb{N}$. Even if $x$ is not LR, there exists a constant $C$ such that $p_{n}(x) \leq C n$ for all $n \in \mathbb{N}$. This is a consequence of the following proposition.

Proposition 2.1. Let $A$ be a finite alphabet, a be a letter of $A$ and $\left(\sigma_{n}: A_{n+1} \rightarrow\right.$ $A_{n}^{+} ; n \in \mathbb{N}$ ) be a sequence of morphisms such that $A_{n} \subset A$ for all $n \in \mathbb{N}, a \in \cap_{n \in \mathbb{N}} A_{n}$ and

$$
y=\lim _{n \rightarrow+\infty} \sigma_{0} \sigma_{1} \cdots \sigma_{n}(\text { aaa } \ldots) .
$$

Suppose moreover $\inf _{c \in A_{n+1}}\left|\sigma_{0} \sigma_{1} \cdots \sigma_{n}(c)\right|$ tends to $+\infty$ and there exists a constant $D$ such that

$$
\left|\sigma_{0} \sigma_{1} \cdots \sigma_{n+1}(b)\right| \leq D\left|\sigma_{0} \sigma_{1} \cdots \sigma_{n}(c)\right|
$$

for all $b \in A_{n+2}$ and $c \in A_{n+1}$, and all $n \in \mathbb{N}$. Then $p_{y}(n) \leq D(\operatorname{Card}(A))^{2} n$. 
Proof. This proof follows the lines of the proof of Proposition V.19 in Qu.

Let $n \geq 1$. The sequence $\left(\inf _{c \in A_{k+1}}\left|\sigma_{0} \sigma_{1} \cdots \sigma_{k}(c)\right|\right)_{k \in \mathbb{N}}$ is non-decreasing and tends to $+\infty$, hence there exists $p \in \mathbb{N}$ such that

$$
\inf _{c \in A_{p}}\left|\sigma_{0} \sigma_{1} \cdots \sigma_{p-1}(c)\right| \leq n \leq \inf _{c \in A_{p+1}}\left|\sigma_{0} \sigma_{1} \cdots \sigma_{p}(c)\right| .
$$

From that, every word $w \in L(y)$ of length $n$ has an occurrence $i$ in some $\sigma_{0} \sigma_{1} \cdots \sigma_{p}(b c)$, where $b$ and $c$ are two letters of $A$, with $i \leq\left|\sigma_{0} \sigma_{1} \cdots \sigma_{p}(b)\right|-1$. Consequently

$$
\begin{gathered}
p_{y}(n) \leq(\operatorname{Card}(A))^{2} \sup _{c \in A_{p+1}}\left|\sigma_{0} \sigma_{1} \cdots \sigma_{p}(c)\right| \\
\leq(\operatorname{Card}(A))^{2} D \inf _{c \in A_{p}}\left|\sigma_{0} \sigma_{1} \cdots \sigma_{p-1}(c)\right| \leq D(\operatorname{Card}(A))^{2} n .
\end{gathered}
$$

This ends the proof.

Corollary 2.1. Let $A$ be a finite alphabet, a be a letter of $A, l$ be a positive integer and $\left(\sigma_{n}: A \rightarrow A^{*} ; n \in \mathbb{N}\right)$ be a sequence of morphisms of constant length $l$ and

$$
y=\lim _{n \rightarrow+\infty} \sigma_{0} \sigma_{1} \cdots \sigma_{n}(\text { aaa } \ldots) .
$$

Then $p_{y}(n) \leq l(\operatorname{Card}(A))^{2} n$.

\section{A sufficient Condition for a PRImitive $S$-ADiC SEQUence to Be LR}

This sufficient condition is given in the following lemma and will be used in the sequel.

Lemma 3.1. Let $S$ be a finite set of morphisms. Let $x$ be a primitive $S$-adic sequence generated by $\left(\sigma_{i}: A_{i+1} \longrightarrow A_{i}^{*} ; i \in \mathbb{N}\right)$ and a (with constant $s_{0}$ ). For all $n \in \mathbb{N}$ suppose $\lim _{l \rightarrow+\infty} \sigma_{n} \sigma_{n+1} \cdots \sigma_{l}($ aaa $\ldots)$ exists and call it $x^{(n)}$. Let $D_{n}$ be the largest difference between two consecutive occurrences of a word of length 2 in $x^{(n)}$.

If $\left(D_{n} ; n \in \mathbb{N}\right)$ is bounded then $x$ is LR.

Proof. Let $x=\lim _{n \rightarrow+\infty} \sigma_{0} \sigma_{1} \cdots \sigma_{n}(a a a \ldots)$. It follows from Lemma 7 of [Du] that $x$ is uniformly recurrent. We set $S_{k}=\sigma_{0} \cdots \sigma_{k}$ for all $k \in \mathbb{N}$. Let $u$ be a non-empty word of $L(x)$ such that $|u| \geq \max \left\{\left|S_{s_{0}}(b)\right| ; b \in A_{s_{0}+1}\right\}$, and $v$ be a return word to $u$. We denote by $k_{0}$ the smallest positive integer $k$ such that $|u|<$ $\min \left\{\left|S_{k}(b)\right| ; b \in A_{k+1}\right\}$; we remark that $k_{0} \geq s_{0}+1$. There exists a word of length $2, b c$, of $L\left(x^{\left(k_{0}+1\right)}\right)$ such that $u$ has an occurrence in $S_{k_{0}}(b c)$. The largest difference between two successive occurrences of $b c$ in $x^{\left(k_{0}+1\right)}$ is bounded by $D=\max _{n \in \mathbb{N}} D_{n}$ (which does not depend on $k_{0}$ ), hence we have

$$
\begin{gathered}
|v| \leq D \max \left\{\left|S_{k_{0}}(d)\right| ; d \in A_{k_{0}+1}\right\} \leq D K \min \left\{\left|S_{k_{0}}(d)\right| ; d \in A_{k_{0}+1}\right\} \\
\leq D K \max \left\{\left|S_{k_{0}-1}(d)\right| ; d \in A_{k_{0}}\right\} \min \left\{\left|\sigma_{k_{0}}(d)\right| ; d \in A_{k_{0}+1}\right\} \\
\leq D K^{2} \min \left\{\left|S_{k_{0}-1}(d)\right| ; d \in A_{k_{0}}\right\} \min \left\{\left|\sigma_{k_{0}}(d)\right| ; d \in A_{k_{0}+1}\right\} \\
\leq D K^{2} \min \left\{\left|\sigma_{k_{0}}(d)\right| ; d \in A_{k_{0}+1}\right\}|u|,
\end{gathered}
$$

where $K$ is the constant given by Lemma 8 of $\mathrm{Du}$, i.e., $K$ is such that for all integers $r, s$ with $s-r \geq s_{0}+1$ and all $b, c$ of $A_{s+1}$ we have $\left|\sigma_{r} \cdots \sigma_{s}(b)\right| \leq K\left|\sigma_{r} \cdots \sigma_{s}(c)\right|$. We set $M=D K^{2} \max \left\{\left|\sigma_{i}(d)\right| ; i \in \mathbb{N}, d \in A_{i+1}\right\}$. For all $u$ of $L(x)$ greater than $\max \left\{\left|S_{s_{0}}(b)\right| ; b \in A_{s_{0}+1}\right\}$ and all $v$ in $\mathcal{R}_{u}$ we have $|v| \leq M|u|$. Hence $x$ is LR. 


\section{A NeCESSARy AND SUfficient CONDition to Be LR}

In the original proof of Proposition 6 in $[\mathrm{Du}$ ] we use the notion of return word. In the proof of Proposition 1.1 we will do the same but we will use an extension of this notion which was defined in [DHS. We will take a sequence $x$ belonging to $X$ and, using these "new" return words, we will show that $x^{+}=x_{[0,+\infty)}$ is a primitive and proper $S$-adic sequence. The subshift $(X, T)$ being minimal we see that it is generated by $x^{+}$and, consequently, $(X, T)$ is a primitive and proper $S$-adic subshift.

Let $A$ be a finite alphabet, $x \in A^{\mathbb{Z}}$, and, $u$ and $v$ two words of $A^{*}$. We say $w \in A^{*}$ is a return word to $u . v$ in $x$ if there exist two consecutive occurrences $j, k$ of $u v$ in $x$ such that $w=x_{[j+|u|, k+|u|)}$. It is immediate to check that a word $w \in A^{+}$ is a return word to $u . v$ in $x$ if and only if:

1) $u w v$ has an occurrence in $x$, and

2) $v$ is a prefix of $w v$ and $u$ is a suffix of $u w$, and

3) the word $u w v$ contains exactly two occurrences of the word $u v$.

We denote by $\mathcal{R}_{x, u . v}$ the set of return words to $u . v$ in $x$. If $u$ is the empty word $\epsilon$ then the return words to u.v are the return words to $v$ defined in $\mathrm{Du}$ and we set $\mathcal{R}_{x, u . v}=\mathcal{R}_{x, v}$. The return words to $u . v$ are different from the return words to $\epsilon . u v$ but we have $\# \mathcal{R}_{x, u . v}=\# \mathcal{R}_{x, \epsilon . u v}=\# \mathcal{R}_{x, u v}$.

We suppose now that $x$ is a uniformly recurrent sequence. It is easy to see that for all $u, v \in L(x)$ the set $\mathcal{R}_{x, u . v}$ is finite. It will be convenient to label the return words. We enumerate the elements $w$ of $\mathcal{R}_{x, u . v}$ in the order of the first appearance of $u w v$ in $x_{[-|u|,+\infty)}$. This defines a bijective map $\Theta_{x, u . v}: R_{x, u . v} \rightarrow \mathcal{R}_{x, u . v} \subset A^{+}$ where $R_{x, u . v}=\left\{1, \ldots, \# \mathcal{R}_{x, u . v}\right\}: u \Theta_{x, u . v}(k) v$ is the $k$-th word of the type $u w v$, $w \in \mathcal{R}_{x, u . v}$, appearing in $x_{[-|u|,+\infty)}$.

We consider $R_{x, u . v}$ as an alphabet. The map $\Theta_{x, u . v}$ defines a morphism from $R_{x, u . v}$ to $A^{*}$ and the set $\Theta_{x, u . v}\left(R_{x, u . v}^{*}\right)$ consists of all concatenations of return words to $u . v$.

The following proposition is important in the proof of Proposition 1.1

Proposition 4.1 ([DHS]). The map $\Theta_{x, u . v}: R_{x, u . v}^{+} \rightarrow A^{+}$is one to one.

Proof of Proposition 1.1. Let $S$ be a finite set of proper morphisms and suppose $(X, T)$ is a primitive $S$-adic subshift generated by $\left(\sigma_{i}: A_{i+1} \rightarrow A_{i}^{*} ; i \in \mathbb{N}\right) \in S^{\mathbb{N}}$ and $a$ (with constant $s_{0}$ ). Let

$$
x=\lim _{n \rightarrow+\infty} \sigma_{0} \sigma_{1} \cdots \sigma_{n}(\text { aaa } \ldots) .
$$

We prove that $x$ is LR and consequently that the subshift it generates is LR.

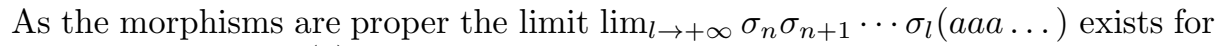
all $n \in \mathbb{N}$. We call it $x^{(n)}$ and we define $D_{n}$ as in Lemma 3.1.

The composition of two proper morphism is again proper. Consequently, from the primitivity, we can suppose that $s_{0}=0$ and that for all $n \in \mathbb{N}$, all $a \in A_{n+1}$ and all $b \in A_{n}$ the letter $b$ appears in $\sigma_{n}(a)$.

Let $n \in \mathbb{N}$ and set $\tau=\sigma_{n} \sigma_{n+1}$. It is a proper substitution. Let $l$ and $r$ be respectively the first and the last letter of the images of $\sigma_{n}$. Let $y$ be a onesided sequence of $A_{n+2}^{\mathbb{N}}$ and $z=\sigma_{n} \sigma_{n+1}(y)$. The words of length 2 having an occurrence in $z$ are exactly the words of length 2 having an occurrence in some $\sigma_{n}(e), e \in A_{n+1}$, and the word $r l$. On the other hand the letters of $\sigma_{n+1}(y)$ appear 
with gaps bounded by $K_{n}=2 \max \left\{\left|\sigma_{n+1}(e)\right| ; e \in A_{n+2}\right\}$. Consequently the words of length 2 of $z$ appear with gaps bounded by $K_{n} \max \left\{\left|\sigma_{n}(e)\right| ; e \in A_{n+1}\right\}$ and, a fortiori, $D_{n} \leq K_{n} \max \left\{\left|\sigma_{n}(e)\right| ; e \in A_{n+1}\right\}$ for all $n \in \mathbb{N}$. Moreover $S$ being finite the sequence $\left(D_{n} ; n \in \mathbb{N}\right)$ is bounded. Lemma 3.1 implies $(X, T)$ is LR.

We suppose now that $(X, T)$ is LR. The periodic case is trivial hence we suppose that $(X, T)$ is not periodic. From Proposition $5 \mathrm{in}[\mathrm{Du}$ there exists $K \geq 2$ such that

$$
(\forall u \in L(X))\left(\forall w \in \mathcal{R}_{u}\right)(|u| / K \leq|w| \leq K|u|),
$$

We set $\alpha=K^{2}(K+1)$. Let $x=\left(x_{n} ; n \in \mathbb{Z}\right)$ be an element of $X$. It suffices to prove that $x_{[0,+\infty)}$ is a primitive and proper $S$-adic sequence.

For each non-negative integer $n$ we set $u_{n}=x_{-\alpha^{n}} \cdots x_{-2} x_{-1}, v_{n}=x_{0} x_{1} \cdots x_{\alpha^{n}-1}$, $\mathcal{R}_{n}=\mathcal{R}_{x, u_{n} . v_{n}}, R_{n}=R_{x, u_{n} . v_{n}}$ and $\Theta_{n}=\Theta_{x, u_{n} . v_{n}}$. Let $n$ be a positive integer and $w$ be a return word to $u_{n} \cdot v_{n}$. The word $w$ is a concatenation of return words to $u_{n-1} \cdot v_{n-1}$. The map $\Theta_{n-1}$ being one to one (Proposition 4.1), this induces a map $\lambda_{n}$ from $R_{n}$ to $R_{n-1}^{*}$ defined by $\Theta_{n-1} \lambda_{n}=\Theta_{n}$. We set $\lambda_{0}=\Theta_{0}$. For each letter $b$ of $R_{n}$ we have $\left|\Theta_{n-1} \lambda_{n}(b)\right| \leq K\left|u_{n} v_{n}\right|=2 K \alpha^{n}$. Moreover each element of $\mathcal{R}_{n-1}$ is greater than $\left(2 \alpha^{n-1}\right) / K$ hence

$$
\left|\lambda_{n}(b)\right| \leq \frac{K^{2} \alpha^{n}}{\alpha^{n-1}}=\alpha K^{2} .
$$

By Proposition 5 of $\left[\mathrm{Du}\right.$ ] we have $\# R_{n}=\# \mathcal{R}_{x, u_{n} v_{n}} \leq K(K+1)^{2}$, consequently the set $M=\left\{\lambda_{n} ; n \in \mathbb{N}\right\}$ is finite. The definition of $R_{n}$ implies that $\Theta_{n}(1) x_{0} x_{1} \cdots x_{\alpha^{n}-1}$ is a prefix of $x_{[0,+\infty)}$ for all $n \in \mathbb{N}$ and $\lambda_{0} \lambda_{1} \cdots \lambda_{n}(1)=\Theta_{n}(1)$. Proposition 4 of [Du implies that the length of $\Theta_{n}(1)$ tends to infinity with $n$ and

$$
x=\lim _{n \rightarrow+\infty} \lambda_{0} \lambda_{1} \cdots \lambda_{n}(11 \cdots) .
$$

Let $n$ be an integer greater than 1 . Each word of length $2 K \alpha^{n}$ has an occurrence in each word of length $2 K(K+1) \alpha^{n}$ (Proposition 5 of $\mathrm{Du}_{\text {] }}$ ). Hence each element of $\mathcal{R}_{n}$ has an occurrence in each word of length $2 K(K+1) \alpha^{n}$. Let $w$ be an element of $\mathcal{R}_{n+1}$, we have $|w| \geq 2 \alpha^{n+1} / K=2 K(K+1) \alpha^{n}$. Therefore each element of $\mathcal{R}_{n}$ has an occurrence in each element of $\mathcal{R}_{n+1}$. It means that if $b$ belongs to $R_{n+1}$ then each letter of $R_{n}$ has an occurrence in $\lambda_{n+1}(b)$. Hence $x$ is a primitive $S$-adic sequence.

It remains to show each $\lambda_{n}$ is proper. Let $w$ be a return word of $\mathcal{R}_{n}$. The word $w v_{n-1}$ is a concatenation of return words to $v_{n-1}$. Let $p \in \mathcal{R}_{n-1}$ be such that $p v_{n-1}$ is a prefix of $w v_{n-1}$ and consequently of $w v_{n}$. We know $v_{n}$ is also a prefix of $w v_{n}$ and

$$
\left|v_{n}\right|=\alpha^{n}=\alpha^{n-1}\left(K^{3}+K^{2}\right) \geq(2 K+1) \alpha^{n-1} \geq|p|+\left|v_{n-1}\right| .
$$

Consequently $p v_{n-1}$ is a prefix of $v_{n}$. Let $l \in R_{n-1}$ be such that $\Theta_{n-1}(l)=p$. Then $l$ is the first letter of $\lambda_{n}(c)$ for all $c \in R_{n}$.

In the same way there exists $s \in \mathcal{R}_{n-1}$ and $r \in R_{n-1}$ such that $\Theta_{n-1}(r)=s$ and $u_{n-1} s$ is a suffix of $u_{n}$. Hence $r$ is the last letter of $\lambda_{n}(c)$ for all $c \in R_{n}$ and $\lambda_{n}$ is proper.

\section{LR STURMian SEQUENCES}

We give a correct proof of the next proposition (which is stated in Du ) because the original proof used Proposition 6 in [Du]. 
Proposition 5.1. A sturmian subshift $\left(\Omega_{\alpha}, T\right)$ is LR if and only if the coefficients of the continued fraction of $\alpha$ are bounded.

Proof. Let $0<\alpha<1$ be an irrational real number and $\left[0: i_{1}+1, i_{2}, i_{3}, \ldots\right]$ be its continued fraction. From Proposition 9 in $[\mathrm{Du}]$ we know that $\Omega_{\alpha}=\Omega(x)$ where

$$
x=\lim _{k \rightarrow+\infty} \tau^{i_{1}} \sigma^{i_{2}} \tau^{i_{3}} \sigma^{i_{4}} \cdots \tau^{i_{2 k-1}} \sigma^{i_{2 k}}(00 \cdots),
$$

$\tau(0)=0, \tau(1)=10, \sigma(0)=01$ and $\sigma(1)=1$. We just have to prove that if the coefficients of the continued fraction of $\alpha$ are bounded then the sequence $x$ is LR. The other part of the proof is in $\mathrm{Du}$ and do not use Proposition 6 in $\mathrm{Du}$.

Let $i, j$ and $k$ be in $\mathbb{N}^{*}$. We have

$$
\tau^{i} \circ \sigma^{j} \circ \tau^{k}(0)=0\left(10^{i}\right)^{j} \text { and } \tau^{i} \circ \sigma^{j} \circ \tau^{k}(1)=10^{i}\left(0\left(10^{i}\right)^{j}\right)^{k} .
$$

Consequently if $x$ belongs to $\{0,1\}^{\mathbb{N}}$ then the set of the words of length 2 having an occurrence in $y=\tau^{i} \circ \sigma^{j} \circ \tau^{k}(x)$ is $\{00,01,10\}$. Moreover the difference of two successive occurrences of 01 (resp. 10) in $y$ is less than $i+2$ (resp. $i+2$ ), and, the difference between two successive occurrences of 00 in $y$ is less than $2 j+3$ if $i=1$ and less than 3 if $i \geq 2$. Consequently the difference between two successive occurrences of a word of length 2 in $y$ is bounded by $2 \max \{i, j, k\}+3$.

The same bound can be found for $\sigma^{i} \circ \tau^{j} \circ \sigma^{k}$.

For all $n \in \mathbb{N}$, let $x^{(n)}$ and $D_{n}$ be defined as in Lemma 3.1. Hence, if the sequence $\left(i_{n} ; n \in \mathbb{N}\right)$ is bounded by $K$, then $D_{n}$ is bounded by $2 K+3$ for all $n \in \mathbb{N}$. Lemma 3.1 ends the proof.

Acknowledgements. I would like to thank Krzysztof Wargan who pointed out the mistake in the proof of Proposition 6 in $\mathrm{Du}$.

\section{REFERENCES}

[Du] F. Durand, Linearly recurrent subshifts have a finite number of non-periodic subshift factors, Ergodic Theory and Dynamical Systems 20 (2000), 1061-1078.

[DHS] F. Durand, B. Host, C. Skau, Substitutions, Bratteli diagrams and dimension groups, Ergodic Theory and Dynamical Systems 19 (1999), 953-993.

[Qu] M. Queffélec, Substitution Dynamical Systems-Spectral Analysis, Lecture Notes in Math. vol. 1294 (1987).

Laboratoire Amiénois de Mathématiques Fondamentales et Appliquées, CNRS-UmR 6140, Université de Picardie Jules Verne, 33 rue Saint Leu, 80039 Amiens Cedex 01, FRANCE

E-mail address: fabien.durand@u-picardie.fr 